\section{Isis takes on myotonic dystrophy}

\section{By Lev Osherovich, Senior Writer}

Researchers at the University of Rochester and Isis Pharmaceuticals Inc. have come up with an efficient way to treat symptoms of myotonic dystrophy type 1 in mice using systemically delivered antisense oligonucleotides. ${ }^{1}$ The finding has fueled an early stage development deal between Isis and Biogen Idec Inc.

Myotonic dystrophy type 1 (DM1) is a dominantly inherited condition that causes progressive muscle weakening and is the most common type of muscular dystrophy, affecting about 1 in 8,000 people. It is caused by spontaneous expansion of a trinucleotide repeat sequence in an untranslated region of the mRNA encoding dystrophia mytonica protein kinase (DMPK; DM1). The resulting transcript is abnormally large and interferes with the activity of nuclear proteins that are critical for normal muscle function.

In severe cases, the disease causes paralysis and respiratory problems. There are no disease-modifying or symptomatic therapies for DM1, leaving palliative physical therapy as the only option for patients.

Prior cell culture and mouse studies by the Rochester team and other academics have suggested that eliminating trinucleotide-expanded DMPK transcripts could alleviate DM $1 .^{2}$

Indeed, the Rochester team, led by Professor of Neurology Charles Thornton, had previously shown in a mouse model of DM1 that disrupting the structure of the expanded RNA sequence that causes DM1 halted the progression of disease and reversed muscle weakening. ${ }^{3}$

Despite these positive data, eliminating the toxic transcript that causes DM1 has been challenging in vivo. The most straightforward approach to targeting the transcript is to use a sequence-specific antisense oligonucleotide (ASO). However, it is difficult to knock down genes in muscle cells because high metabolic and transcriptional activity in these cells leads to high levels of many transcripts.

As a result, prior efforts to knock down repeat-expanded DMPK transcripts in DM1 mice required a complex, high-dose regimen of injections into affected muscles. ${ }^{4}$

In the new study, Thornton and collaborators at Isis led by C. Frank Bennett, SVP of research, set out to solve the delivery and dosing challenges.

The team constructed an ASO that hits its target in a variety of muscle tissues after a single subcutaneous injection. Moreover, the ASO persisted within the muscle cells and prevented further RNA buildup months after the initial treatment.

"This is one of the more robust demonstrations of using antisense oligonucleotides to target muscle tissue," said Bennett. "Historically we've thought of muscle tissue as being on the lower end of responsiveness to antisense technology. Here we show that doses that could be used in a clinical setting can actually be effective."

\section{Single shot}

Bennett and Thornton first ran cell culture screens for ASOs that knocked down the expression of a model transcript bearing the DM1associated trinucleotide repeats. The most potent hits were then tested in a mouse model expressing a model transcript with the DM1-associated trinucleotide repeats.

In cell culture and mice, ASOs decreased levels of the toxic RNA compared with a scrambled-sequence control ASO and alleviated the nuclear accumulation of proteins trapped by the aberrant transcript.

Mice receiving a single subcutaneous injection of the most potent ASOs showed better function in a variety of muscle groups than animals given scrambled control. The effect of the ASOs on muscle strength lasted for months after dosing ceased, suggesting that the molecule remains in the nucleus for a lengthy period.

Thornton said subcutaneous injection of the ASOs proved surprisingly effective and represented a leap beyond prior tissue-specific RNA knockdown strategies tried by his team.

"This was a whole-body treatment," said Thornton. "All of the muscles of the mouse were corrected by a single injection into the skin. This is the biggest difference compared with prior approaches."

He noted that DM1 affects a range of muscle tissue groups and displays an unpredictable course of progression in many patients. Rather than try to treat it in each "This is one of the more robust demonstrations of using antisense oligonucleotides to target muscle tissue. [...] Here we show that doses that could be used in a clinical setting can actually be effective."

-C. Frank Bennett, Isis Pharmaceuticals Inc. said it would be better to have a systemic therapy.

The high potency of the ASOs may be due to the manner in which the antisense molecules find and destroy their target, said Bennett.

The company's ASOs work by flagging target transcripts for destruction by RNase $\mathrm{H}$, a cellular enzyme that ordinarily helps degrade excess or abnormal mRNAs.

"Our drugs work through RNase $\mathrm{H}$, which recognizes an RNADNA heteroduplex," said Bennett. "We get cleavage of the target RNA by RNase H."

Bennett said that RNase $\mathrm{H}$ is especially abundant in the nuclei of muscle cells. Because the DM1-associated transcript also accumulates in the nucleus, all that is needed to trigger the RNA's destruction is a few molecules of ASO to help connect the RNase $\mathrm{H}$ enzyme to its target.

Thornton previously used morpholinos, a class of synthetic RNAbinding molecule, to prevent the toxic effects of trinucleotide-expanded RNA. ${ }^{3}$ Morpholinos are RNA-like molecules that bind to sequence- 


\section{ANALYSIS}

\section{TARGETS \& MECHANISMS}

matched nucleic acid targets but do not necessarily cause the degradation of their targets.

He said ASOs are likely a better therapeutic class.

"By changing our strategy from trying to block the toxic RNA and insulating it to getting rid of it by cleavage, we gained a huge potency advance," said Thornton. "Now even the relatively small amount of material that goes into muscle cells is effective."

Results were published in Nature.

\section{Dollars and antisense}

In June, Isis granted Biogen rights to ASO therapeutics for DM1 in exchange for $\$ 12$ million up front and up to $\$ 59$ million in preclinical and clinical milestones.

Bennett said Isis is now screening for variants of the ASOs with even greater potency than the ASOs used in the study. He did not disclose a timetable for clinical development of the compounds.

Meanwhile, Dutch biotech Prosensa B.V. is pursuing its own ASO strategy for DM1. In 2009, Thornton collaborated with Prosensa and researchers at Radboud University Nijmegen Medical Centre to show that the biotech's ASOs could reduce DM1-like pathology in the same mouse model used in the Isis study.

Compared with Isis' ASOs, Prosensa's technology uses a different backbone chemistry and relies on other cellular nucleases besides RNase $\mathrm{H}$ to cleave target mRNAs, said CBO and SVP of business development Luc Dochez.

Dochez said it is too early to say how the two approaches stack up against one another and that a real comparison "would require PK/PD studies in humans."

To maximize the activity of Prosensa's ASOs in muscle tissues, Dochez said the company is designing a preclinical candidate ASO fused to a muscle cell-targeting peptide. The program and its lead product, PRO135, is available for out-licensing or partnering.

Dochez noted that some patients with DM1 exhibit neurological symptoms, so it may also be desirable to hit expanded DMPK transcripts in the CNS, though it is not yet clear how to do so.

"A muscle-targeting peptide could be a way to overcome the challenges of systemic delivery," said Dochez. "With muscle-targeting delivery, we could address the myotonia in the muscle, but there's also a CNS component in the more severe forms of the disease, which poses an additional challenge. But even addressing part of the disease could be a big leap."

Isis has filed for patents covering the findings described in the Nature paper.

Osherovich, L. SciBX 5(31); doi:10.1038/scibx.2012.806

Published online Aug. 9, 2012

\section{REFERENCES}

1. Wheeler, T.M. et al. Nature; published online Aug. 2, 2012; doi:10.1038/nature11362

Contact: Charles Thornton, University of Rochester, Rochester, N.Y. e-mail: charles_thornton@urmc.rochester.edu

2. Muntoni, F. \& Wood, M.J. Nat. Rev. Drug Discov. 10, 621-637 (2011)

3. Wheeler, T.M. et al. Science 325, 336-339 (2009)

4. Mulders, S.A.M. et al. Proc. Natl. Acad. Sci. USA 106, 13915-13920 (2009)

COMPANIES AND INSTITUTIONS MENTIONED

Biogen Idec Inc. (NASDAQ:BIIB), Weston, Mass.

Isis Pharmaceuticals Inc. (NASDAQ:ISIS), Carlsbad, Calif.

Prosensa B.V., Leiden, the Netherlands

Radboud University Nijmegen Medical Centre, Nijmegen, the Netherlands

University of Rochester, Rochester, N.Y. 